\title{
BROTE DE INFECCIÓN POR NOROVIRUS DEL GRUPO II EN UN CENTRO DE ENTRENAMIENTO MILITAR, PERÚ 2013
}

\author{
Mariana Ramos ${ }^{1, a, i}$, Giselle Soto ${ }^{1, a, j,}$ Luis Loayza ${ }^{2, b}, R^{2}$ ubén Valle ${ }^{1, a, k}$, Giannina Luna ${ }^{1, c, l}$, Sofia Gonzalez $z^{3, d}$, \\ Jose Quispe ${ }^{2, e}$, Maria Silva ${ }^{1, f, m}$, Juan Wong ${ }^{1}$, Daniel G. Bausch ${ }^{1, g, h}$
}

\begin{abstract}
RESUMEN
Reportamos un brote de norovirus (NoV) ocurrido en un centro de entrenamiento militar peruano. Trece muestras de heces tomadas de casos iniciales resultaron positivas a NoV GII por RT-PCR. Se realizó un estudio caso-control para determinar factores asociados a la infección. Se identificó 164 casos probables (tasa de ataque clínica: 45,2\%). El único factor asociado a la infección fue el "haber compartido habitación con un caso" (ORa=1,7; IC95\% 1,1 a 2,7). Los hallazgos epidemiológicos sugieren que el virus fue propagado persona a persona. Futuras investigaciones de brotes de diarrea en el Perú deberían considerar al NoV como un agente etiológico.
\end{abstract}

Palabras clave: Norovirus; Brotes; Personal militar (fuente: DeCS BIREME).

\section{OUTBREAK OF NOROVIRUS GROUP II IN A MILITARY TRAINING CENTER, PERU 2013}

\begin{abstract}
We report an outbreak of norovirus (NoV) which occurred at a military training center in Peru. Thirteen stool samples that were taken from initial cases turned out positive for NoV GIl by RT-PCR. A case-control study was performed to determine factors associated with infection.164 probable cases (45.2\% clinical attack rate) were identified. The only factor associated with infection was "having shared room with a case" (ORa $=1.7 ; 95 \% \mathrm{Cl} 1.1$ to 2.7$)$. Epidemiological findings suggest that the virus was spread person to person. Future investigations of outbreaks of diarrhea in Peru should consider the NoV as an etiologic agent.
\end{abstract}

Key words: Norovirus; Disease outbreaks; Military personnel (source: MeSH NLM).

\section{INTRODUCCIÓN}

Las gastroenteritis virales se presentan como enfermedades endémicas o epidémicas en infantes, niños y adultos ${ }^{(1)}$. Muchos virus, entre ellos rotavirus, adenovirus entérico, astrovirus y calicivirus causan diarrea severa en niños. El norovirus, por otro lado, suele afectar a población adulta ${ }^{(2)}$. El norovirus (NoV) es la causa más frecuente de gastroenteritis a nivel mundial siendo responsable de alrededor de $50 \%$ de los brotes de esta condición en países desarrollados ${ }^{(1)}$. En países de clima templado la mayor incidencia ocurre en los meses fríos del año ${ }^{(3)}$. Su tiempo de incubación es de 24 a 72 h y se caracteriza clínicamente por inicio agudo de nauseas, vómitos, dolor abdominal y diarrea no disentérica todo lo cual puede durar entre 2 a 4 días en personas inmunocompetentes ${ }^{(1,4)}$. Los vómitos son particularmente frecuentes, presentándose en más del $30 \%$ de los casos $^{(4)}$.

La gastroenteritis por NoV suele presentarse en forma epidémica en lugares cerrados como hospitales, cruceros, centros de reposo o cuarteles (1). Puede transmitirse tanto por vía fecal-oral como persona a persona, a través de fómites o por aerosolización de partículas de vómito o heces ${ }^{(1)}$. Algunas características del NoV facilitan su dispersión durante un brote, entre ellas su baja dosis infectiva ${ }^{(5)}$; la secreción viral larga por

\footnotetext{
Centro de Investigación de Enfermedades Tropicales de la Marina de EE. UU., Lima, Perú.

Dirección de Salud de la Marina de Guerra del Perú, Lima, Perú.

Centro Médico Naval “CMST”, Lima, Perú

Médico cirujano; ${ }^{\mathrm{b}}$ odontólogo pediatra; ${ }^{\mathrm{c}}$ bióloga; ${ }^{\mathrm{d}}$ médico infectóloga; ${ }^{\mathrm{e}}$ enfermero naval; ${ }^{\mathrm{f}}$ médico veterinario; ${ }^{\mathrm{g}}$ médico tropicalista; ${ }^{\mathrm{h}}$ máster en Salud Pública; ${ }^{i}$ maestría en Epidemiología con mención en métodos cuantitativos; ' maestría en Salud Pública; ${ }^{k}$ maestría en Investigación Epidemiológica; ${ }^{1}$ maestría en Control de Enfermedades Infecciosas; ${ }^{\mathrm{m}} \mathrm{PhD}$

Recibido: 11-07-14 Aprobado: 11-02-15
}

Citar como: Ramos M, Soto G, Loayza L, Valle R, Luna G, Gonzalez S, et al. Brote de infección por norovirus del grupo II en un Centro de Entrenamiento Militar, Perú 2013. Rev Peru Med Exp Salud Publica. 2015;32(1):87-92. 
parte de los casos, incluso aquellos asintomáticos; la estabilidad del virus en concentraciones relativamente altas de cloro y a un amplio rango de temperaturas ${ }^{(6)}$; y la ausencia de inmunidad a largo plazo ${ }^{(1)}$.

Las poblaciones militares son particularmente susceptibles a brotes por NoV (7-9) debido al hacinamiento en el que conviven, los malos hábitos higiénicos y a la constante manipulación de grandes cantidades de alimentos ${ }^{(7)}$. Asimismo, dichos brotes pueden comprometer la efectividad operativa de los individuos afectados ${ }^{(10)}$. Se han reportado brotes de NoV en estas poblaciones en diversas partes del mundo, muchos de ellos se iniciaron debido al consumo de productos marinos, pero se perpetuaron como consecuencia de la capacidad del virus de transmitirse persona a persona $(8,11,12)$.

El 3 de octubre de 2013, el sistema de vigilancia electrónica Alerta DISAMAR de la Marina de Guerra del Perú recibió una notificación de incremento de casos de enfermedad diarreica aguda en uno de sus centros de entrenamiento ubicado en Lima. Por tal motivo, se llevó a cabo una investigación con la finalidad de identificar el mecanismo de transmisión y el agente etiológico del brote. A continuación presentamos los hallazgos de la investigación del brote así como las medidas de control implementadas para cortar la cadena de transmisión.

\section{EL ESTUDIO}

Un equipo de primera respuesta formado por epidemiólogos y otro personal de salud del Centro de Investigación de Enfermedades Tropicales de la Marina de EE. UU. (NAMRU-6), Lima, Perú y de la Marina de Guerra del Perú llegó al lugar el 4 de octubre de 2013. La investigación constó de dos fases: una descriptiva que permitió caracterizar el brote y generar hipótesis acerca de la fuente de contaminación y etiología; y una analítica, en la que se usó un diseño de caso-control para probar dichas hipótesis. La primera aproximación constó de entrevistas a personal clave, como médicos a cargo del manejo de los pacientes bajo observación. Del mismo modo, se revisó del libro de atenciones del policlínico del centro de entrenamiento militar con una ventana de 10 días previos al inicio del brote. El punto de corte de la búsqueda de casos corresponde a 3 periodos de incubación máximos (1 a 3 días) de la mayoría de patógenos virales y bacterianos que afectan el tracto gastrointestinal, y se usó con la finalidad de identificar al caso primario. Se definió como caso probable a todo "paciente procedente del centro de instrucción que hubiera presentado diarrea, vómitos o dolor abdominal desde el 20 de septiembre de 2013". Se consideró como caso confirmado a todo aquel caso probable positivo para norovirus en la prueba de reacción en cadena de polimerasa en tiempo real (RT-PCR). Durante esta primera fase se recolectó 5 muestras de heces de pacientes en fase aguda de la enfermedad para estudio de bacterias y virus, y 15 muestras ambientales que incluyeron agua, hisopados de trapos de cocina, lavadero, licuadoras, tablas de picar, platos e hisopado de manos a manipuladores de alimentos. Todas las muestras fueron procesadas y analizadas en los laboratorios del NAMRU-6. Para el estudio de bacterias se usó técnicas de cultivo convencional. El diagnóstico de norovirus GI y GII se realizó mediante la técnica de reacción en cadena de la polimerasa en tiempo real doble haciendo uso de dos sets de cebadores diseñados para amplificar el área comprendida entre las regiones abiertas de lectura $1 \mathrm{y}$ 2 (ORF1 - ORF2).

Tras los primeros hallazgos del estudio descriptivo, se realizó un estudio caso-control para identificar factores asociados a la enfermedad durante el brote. Este estudio se llevó a cabo 2 semanas después de reportado el caso índice, y el periodo de búsqueda se amplió hasta 60 días previos al reporte, ante la sospecha de la presencia de casos aun más tempranos que no buscaron atención médica y que, como consecuencia, no figuraban en los registros. La definición de caso fue la misma usada para la fase descriptiva, mientras que los controles fueron todos aquellos estudiantes que no presentaron diarrea, vómitos o dolor abdominal durante el periodo establecido. Las variables evaluadas incluyeron características clínicas, edad, sexo, año de estudios, contacto con personas sintomáticas, hábitos de lavado de manos y una historia detallada de consumo de alimentos.

El cuestionario aplicado fue anónimo y autoadministrado. Se solicitó, además, una muestra de heces a todos los participantes para evaluar casos asintomáticos o no reportados. EI Comité de Ética del NAMRU-6 fue notificado acerca del brote. Por tratarse de una intervención de salud pública, esta investigación no requirió pasar por un panel revisor. Para el análisis de datos se calculó Odds Ratios (OR) crudos y ajustados e intervalos de confianza al 95\% usando análisis de regresión logística múltiple (stepwise) con STATA $13.0^{\circledR}$. Aquellas variables con un $\mathrm{p}<0,1$ ingresaron al modelo multivariado.

\section{HALLAZGOS}

La población de estudiantes en el centro de entrenamiento fue de 450 . 


\section{Primera etapa: estudio descriptivo}

Se identificó 83 casos probables, de ellos 67 (80,7\%) estuvieron disponibles para la evaluación. La mayor parte $(90,9 \%)$ fueron estudiantes (tasa de ataque clínica $18,4 \%$ ). La mediana de la edad fue 20 años (rango: $17,5,21,0)$ y el $92,5 \%$ fueron de sexo masculino. El caso índice inicio sus síntomas el 29 de septiembre de 2013. Todos los casos fueron manejados ambulatoriamente. El análisis bacteriológico de las 15 muestras ambientales así como de las cinco muestras de heces fue negativo para Salmonella, Shigella, Vibrio, Campylobacter, Yersinia enterocolitica, Staphilococcus aureus, Bacilus cereus y Escherichia coli enterotoxigénica. Cuatro de las cinco muestras de heces resultaron positivas para NoV del Grupo II. La muestra restante no fue procesada para NoV por ser insuficiente.

\section{Segunda etapa: estudio caso-control}

En el estudio caso-control se entrevistó a 363 de los 450 estudiantes del centro $(80,7 \%)$, de los cuales 164 fueron casos y 199 controles $(1: 1,2)$. Asimismo, se obtuvo 79 muestras de heces $(21,8 \%)$. Con la inclusión de estos casos, la tasa de ataque clínica al culminar el brote fue tres veces mayor que la detectada en el estudio descriptivo $(45,2 \%$ vs $18,4 \%)$ y la mediana de la duración de los síntomas fue de 48 h (rango: 2472 ). El dolor abdominal $(82,3 \%)$, el malestar general $(53,3 \%)$ y la diarrea $(54,9 \%)$ fueron los síntomas más frecuentes y más de la tercera parte de los entrevistados $(34,2 \%)$ reportó al menos 1 episodio de vómito. Trece de las 79 muestras de heces colectadas fueron positivas para NoV GII, dos de ellas correspondieron a controles (el primero asintomático y el otro con síndrome febril inespecífico). Se encontró diferencia estadística entre casos y controles en relación a la presencia de NoV en heces $(p=0,02)$. La curva epidémica mostró hasta tres conglomerados de casos, considerando que solo el último de ellos correspondería al brote por coincidir con el tiempo de incubación de NoV. El caso primario inicio síntomas el 21 de septiembre de 2013 (Figura 1). La curva epidémica muestra un patrón consistente con transmisión persona a persona.

El análisis bivariado se muestra en la Tabla 1. Las variables que resultaron asociadas a la infección fueron "el compartir habitación con un caso" (OR=1,7; IC $95 \% 1,1$ a 2,6); "el lavarse las manos después de usar el gimnasio" (OR=0,6; IC 95\% 0,4 a 1,0); y un grupo de alimentos entre los que figuraban la anchoveta (OR=1,7; IC 95\% 1,1 a 2,9); frutas crudas (OR=0,5; IC $95 \% 0,3$ a 0,8); ensalada de frutas (OR=0,6; IC $95 \% 0,4$ a 1,0 ) y ensalada de verduras (OR=0,6; IC $95 \%$ 0,4 a

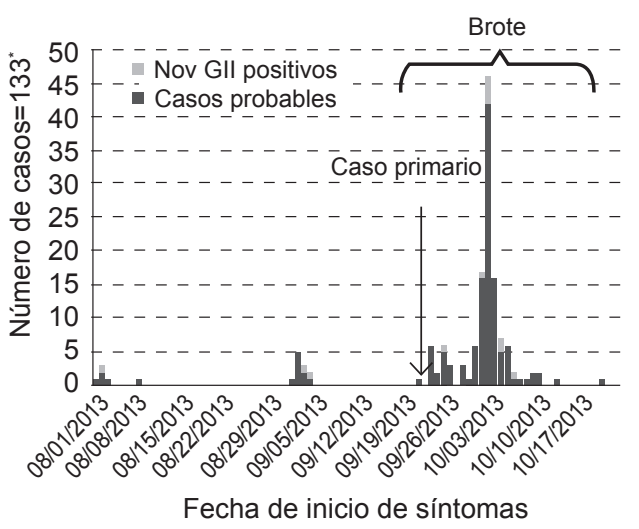

Figura 1. Curva epidémica de los casos probables y confirmados obtenida en el estudio caso-control ${ }^{\star *}$

* 31 de los 164 casos no proporcionaron fecha de inicio de síntomas

** Tiempo de incubación de NoV: 24-72 horas.

0,9). Al realizar el análisis multivariado, solo "el haber compartido habitación con un caso" (ORa=1,6; IC $95 \%$ 1,1 a 2,7 ; valor $p=0,03$ ) permaneció asociado a la enfermedad, ajustando por lavado de manos después del gimnasio, ingesta de anchoveta, frutas, ensalada de frutas y verduras.

\section{DISCUSIÓN}

Este estudio documenta un brote de gran magnitud de enfermedad por NoV en un centro de entrenamiento militar del Perú, con una tasa de ataque clínica notablemente alta $(45,2 \%)$. En comparación, durante las operaciones "Desert Torment" y "Desert Shield" de las Fuerzas Armadas de Estados Unidos, se presentaron múltiples brotes por NoV con tasas de ataque que fluctuaron entre 6 y $13 \%{ }^{(7)}$. Cabe destacar que la tasa de ataque calculada en nuestro estudio no estuvo basada en casos confirmados por laboratorio sino solo por nexo epidemiológico, por lo que es posible que una proporción de dicha cifra se deba a otras etiologías de presentación clínica similar.

A pesar de que los brotes por NoV son reportados frecuentemente en todo el mundo, hay pocos datos del Perú (13-16). En la literatura científica solo figuran dos reportes en poblaciones militares en la región ${ }^{(8,9)}$. En la mayoría de los estudios en el Perú, como en el nuestro, el genotipo GII predominó (13-16). La infección por NoV suele introducirse en la población a través de la vía fecal-oral y perpetuarse en varias generaciones de casos a través de la transmisión persona a persona ${ }^{(7)}$. En este brote, la curva epidémica y las asociaciones significativas con el haber compartido habitación con un caso sugiere que el virus se propagó dentro del centro de instrucción 
Tabla 1. Factores asociados a la enfermedad durante el brote.

\begin{tabular}{|c|c|c|c|c|c|c|c|}
\hline \multirow[b]{2}{*}{ Variable } & \multicolumn{2}{|c|}{ Caso } & \multicolumn{2}{|c|}{ Control } & \multirow{2}{*}{$\mathrm{OR}^{\varepsilon}$} & \multirow{2}{*}{$(\mathrm{IC} 95 \%)^{\epsilon}$} & \multirow{2}{*}{ Valor $p$} \\
\hline & $n=164$ & $\%$ & $n=199$ & $\%$ & & & \\
\hline Edad (mediana, intervalo intercuartil) & $20(19-22)$ & & $20(19-22)$ & & 1,0 & $(0,9-1,1)$ & 0,6 \\
\hline Sexo masculino & 148 & 90,2 & $185 / 198$ & 93,4 & 0,7 & $(0,3-1,4)$ & 0,3 \\
\hline Individuos por habitación (mediana, intervalo intercuartil) & $6(5-6)$ & & $6(5-6)$ & & 0,9 & $(0,7-1,1)$ & 0,8 \\
\hline \multicolumn{8}{|l|}{ Rango } \\
\hline Cadete aspirante & 43 & 26,2 & 47 & 23,6 & 1,1 & $(0,7-1,9)$ & 0,9 \\
\hline Cadete de primer año & 34 & 20,7 & 41 & 20,6 & 1,00 & $(0,6-1,7)$ & \\
\hline Cadete de segundo año & 32 & 19,5 & 36 & 18,1 & 1,1 & $(0,6-1,9)$ & \\
\hline Cadete de tercer año & 23 & 14,0 & 28 & 14,1 & 1,0 & $(0,5-1,8)$ & \\
\hline Cadete de cuarto año & 30 & 18,3 & 45 & 22,6 & 0,8 & $(0,5-1,3)$ & \\
\hline Oficial alumno & 2 & 1,2 & 2 & 1,0 & 1,2 & $(0,2-8,7)$ & \\
\hline \multicolumn{8}{|l|}{ Grupo sanguíneo (casos $n=160$; controles $n=191$ )* } \\
\hline $\mathrm{O}+$ & 104 & 65,0 & 135 & 70,7 & 0,8 & $(0,5-1,2)$ & 0,2 \\
\hline O - & 3 & 1,9 & 4 & 2,1 & 0,9 & $(0,2-4,1)$ & \\
\hline$A+$ & 35 & 21,9 & 30 & 15,7 & 1,5 & $(0,9-2,6)$ & \\
\hline A - & 3 & 1,9 & 0 & 0 & 0 & & \\
\hline$B+$ & 12 & 7,5 & 15 & 7,9 & 1 & $(0,4-2,1)$ & \\
\hline$B-$ & 0 & 0 & 1 & 0,5 & 0 & & \\
\hline$A B+$ & 3 & 1,9 & 6 & 3,1 & 0,6 & $(0,1-2,4)$ & \\
\hline \multicolumn{8}{|l|}{ Contacto } \\
\hline Compartió habitación con un caso & $105 / 156$ & 67,3 & $109 / 197$ & 55,3 & 1,7 & $(1,1-2,7)$ & 0,02 \\
\hline Contacto con caso dentro de la Escuela Militar & $106 / 160$ & 66,3 & $125 / 198$ & 63,1 & 1,1 & $(0,7-1,8)$ & 0,5 \\
\hline Contacto con vómito & 73 & 44,5 & 88 & 44,2 & 1,1 & $(0,7-1,5)$ & 1,0 \\
\hline Contacto con caso fuera de la Escuela Militar & 6 & 3,7 & 6 & 3,1 & 1,2 & $(0,4-3,8)$ & 0,7 \\
\hline \multicolumn{8}{|l|}{ Lavado de manos (casos $n=163$; controles $n=198$ ) } \\
\hline Lavado de manos solo cuando están evidentemente sucias & 126 & 77,3 & 161 & 81,3 & 0,78 & $(0,46-1,30)$ & 0,3 \\
\hline Lavado de manos después de ir al baño & 122 & 74,9 & 148 & 74,8 & 1,0 & $(0,6-1,6)$ & 1,0 \\
\hline Lavado de manos antes de comer & $121 / 162$ & 74,7 & $138 / 197$ & 70,1 & 1,3 & $(0,8-2,0)$ & 0,3 \\
\hline Lavado de manos después de hacer ejercicio & $112 / 161$ & 69,6 & $128 / 197$ & 65,0 & 1,2 & $(0,8-1,9)$ & 0,4 \\
\hline Lavado de manos después de usar el gimnasio & $86 / 160$ & 53,8 & $127 / 197$ & 64,5 & 0,6 & $(0,4-1,0)$ & 0,04 \\
\hline \multicolumn{8}{|l|}{ Alimentos (desde el 23 de setiembre) } \\
\hline Consumo de carne mal cocida & $136 / 157$ & 86,6 & $174 / 196$ & 88,8 & 0,8 & $(0,4-1,6)$ & 0,5 \\
\hline Anchoveta & $133 / 163$ & 81,6 & $142 / 198$ & 71,7 & 1,7 & $(1,1-2,9)$ & 0,03 \\
\hline Pescado & $125 / 163$ & 76,7 & $147 / 197$ & 74,6 & 1,1 & $(0,7-1,8)$ & 0,7 \\
\hline Frutas crudas & $101 / 163$ & 62,0 & $152 / 198$ & 76,8 & 0,5 & $(0,3-0,8)$ & 0,02 \\
\hline Ensalada de frutas & $92 / 163$ & 56,4 & $132 / 198$ & 66,8 & 0,6 & $(0,4-1,0)$ & 0,05 \\
\hline Pachamanca & $91 / 163$ & 55,8 & $114 / 197$ & 57,9 & 0,9 & $(0,6-1,4)$ & 0,7 \\
\hline Mayonesa & $89 / 163$ & 54,6 & $112 / 198$ & 56,6 & 0,9 & $(0,6-1,4)$ & 0,7 \\
\hline Ensalada de verduras & $84 / 162$ & 51,9 & $126 / 198$ & 63,6 & 0,6 & $(0,4-0,9)$ & 0,02 \\
\hline Mariscos & $37 / 163$ & 22,7 & $61 / 198$ & 30,8 & 0,7 & $(0,4-1,1)$ & 0,09 \\
\hline Conchas & $75 / 162$ & 9,3 & $28 / 196$ & 14,3 & 0,6 & $(0,3-1,2)$ & 0,2 \\
\hline \multicolumn{8}{|l|}{ Fuente de agua para consumo (desde el 23 de septiembre) } \\
\hline Agua embotellada & 141 & 86,0 & 175 & 87,9 & 0,8 & $(0,5-1,6)$ & 0,6 \\
\hline Comedor de la escuela & 66 & 40,2 & 96 & 48,2 & 0,7 & $(0,5-1,1)$ & 0,2 \\
\hline Agua de bebederos & 8 & 4,9 & 8 & 4,0 & 1,2 & $(0,4-3,3)$ & 0,7 \\
\hline Otra fuente de agua & 7 & 4,3 & 15 & 7,5 & 0,5 & $(0,2-1,4)$ & 0,2 \\
\hline Consumo de agua de caño sin desinfectar & 31 & 18,9 & $34 / 167$ & 17,3 & 1,11 & $(0,7-1,9)$ & 0,7 \\
\hline Consumo de agua en balde sin desinfectar & $5 / 161$ & 3,1 & $3 / 197$ & 1,5 & 2,07 & $(0,5-8,8)$ & 0,3 \\
\hline Consumo de agua de pozo sin desinfectar & 4 & 2,4 & $3 / 197$ & 1,5 & 1,61 & $(0,4-7,3)$ & 0,5 \\
\hline
\end{tabular}

* El norovirus reconoce los antígenos del grupo sanguíneo como receptores ${ }^{(20)}$

£ Odds ratio

$\epsilon$ Intervalo de confianza al $95 \%$ 
de persona a persona. Si bien en el análisis bivariado, algunos alimentos mostraron asociación, esta no se mantuvo presente en el análisis multivariado por lo que no fue posible asociar el inicio del brote con un alimento en especial. Es probable que la contaminación ambiental con el virus haya contribuido a la transmisión, como ha sido previamente reportado ${ }^{(17)}$.

Debido a limitaciones logísticas no fue posible conseguir muestras de alimentos para análisis. Además, los hisopados de manos de manipuladores de alimentos resultaron negativos para patógenos bacterianos, pero no fueron testeados para virus por no contar con un procedimiento estandarizado. Algunas de las recomendaciones brindadas a las autoridades militares fueron el aislamiento de los casos en un área común; la suspensión de todas las actividades grupales durante la duración del brote; el lavado de manos con agua y jabón especialmente después de acudir al baño y antes de comer; la desinfección frecuente de áreas comunes y superficies de alto tránsito (baños, teléfonos, manijas de puertas, computadoras, equipos de gimnasio etc.) y la eliminación inmediata de material usado para limpiar heces o vómitos ${ }^{(7,18)}$.

El caso primario probablemente fue aquel que inició síntomas el 21 de septiembre; sin embargo, se observaron previamente hasta dos conglomerados pequeños de casos positivos a NoV con duración de síntomas mayor a 45 días. Aunque la excreción de NoV en heces normalmente suele durar 4 a 6 días tras el inicio de síntomas en pacientes inmunocompetentes, se ha observado tiempos de excreción de hasta 30 días ${ }^{(18)}$, hecho que abre la posibilidad de que la introducción del virus causante del brote pudo haber ocurrido por un caso perteneciente a uno de los dos conglomerados precedentes. Teniendo esto en cuenta, es posible que varios casos probables ocurridos antes del brote principal quedaran sin detectar. Estos casos ocultos constituyeron un riesgo para el resto de la población y un reto para el personal de salud. El rol de estos individuos en el contagio fue difícil de evaluar en nuestro estudio debido al diseño de estudio retrospectivo que se realizó.

Brotes investigados en poblaciones militares muestran que existe con frecuencia un patrón de no búsqueda de atención médica ${ }^{(19)}$. Nuestra hipótesis es que esta conducta es consecuencia de una preocupación real o percibida de los sujetos de quedar imposibilitados de salir de descanso al encontrarse bajo observación médica por enfermedad, inclusive solo por síntomas leves. Al quedar hospitalizado de manera obligatoria el paciente perdería el descanso de los fines de semana tras haber permanecido internado en la base de lunes a viernes, lo cual es considerado como un perjuicio grande, al parecer mayor que el de permanecer sintomático durante el entrenamiento diario.
Por esta tendencia de las poblaciones militares de esconderse de detección, hemos encontrado que la mejor manera de establecer tasas de ataque clínica reales y factores de riesgo en estas poblaciones es usando cuestionarios anónimos (19). Sin embargo, la necesidad de mantener el anonimato para obtener cifras reales no permitió relacionar al caso primario con aquellos individuos secretores de largo periodo, lo cual fue una limitación de nuestro estudio. Si bien existen sistemas de detección temprana de casos, como el sistema Alerta DISAMAR de la Marina de Guerra, es recomendable sensibilizar a la población militar en cuanto a las consecuencias potenciales que tendrían para la salud pública el no reportar sus síntomas a tiempo.

En conclusión, se identificó como NoV GII como el agente etiológico del brote, transmitido persona a persona por contacto directo con un caso. La poca disponibilidad de pruebas diagnósticas moleculares y la falta de presupuesto para compra de equipos y capacitación de personal en zonas remotas hace difícil el diagnóstico del NoV en la mayoría de los casos ${ }^{(5,7)}$. Es probable que el NoV sea responsable de gran parte de la carga de enfermedad por gastroenteritis a nivel nacional en el Perú. Recomendamos mayor investigación al respecto e inversión en la implementación y entrenamiento de personal en pruebas de detección en zonas de alto riesgo.

Descargo de responsabilidad: las opiniones vertidas en este artículo son de los autores y no necesariamente reflejan la política oficial o la posición del Departamento de la Marina, Departamento de Defensa, o el Gobierno de los Estados Unidos.

Algunos autores son personal contratado por el gobierno de los Estados Unidos y este trabajo ha sido preparado como parte de sus funciones oficiales.

Agradecimientos: agradecemos al Dr. Hugo Gallo Seminario por su colaboración con el estudio.

Contribuciones de autoría: MR, GS, RB, SG y DB han participado en la concepción y diseño del artículo. MR, GS, LL, $\mathrm{RV}, \mathrm{GL}, \mathrm{JQ}$ y MS participaron en la recolección de resultados. MR, GS, LL, RV, GL y MS analizaron e interpretaron los datos. MR se encargó de la redacción del artículo. GS, LL, $\mathrm{RV}, \mathrm{GL}, \mathrm{SG}, \mathrm{MS}, \mathrm{JW}$ y DB revisaron críticamente el artículo. MR, LL, JQ y JW aportaron pacientes o material de estudio. MR y JW participaron en la obtención del financiamiento. MR brindó asesoría estadística. LL, GL, SG, JQ y JW brindaron asesoría técnica o administrativa. DB aprobó la versión final del artículo.

Fuentes de financiamiento: este trabajo fue financiado por el fondo de la unidad de trabajo número 847705.82000.25GB. B0016.

Conflictos de interés: los autores declaran no tener conflictos de interés. 


\section{REFERENCIAS BIBLIOGRÁFICAS}

1. Patel MM, Hall AJ, Vinjé J, Parashar UD. Noroviruses: a comprehensive review. J Clin Virol. 2009 Jan;44(1):18. doi: 10.1016/j.jcv.2008.10.009.

2. Fontaine O. Viral acute gastroenteritis. En: Heymann DL. Control of Communicable Diseases Manual. 19th ed. Washington, DC: American Public Health Association; 2008.

3. Mounts AW, Ando T, Koopmans M, Bresee JS, Noel J, Glass RI. Cold weather seasonality of gastroenteritis associated with Norwalk-like viruses. J Infect Dis. 2000 May;181 Suppl 2:S284-7.

4. Turcios RM, Widdowson MA, Sulka AC, Mead PS, Glass RI. Reevaluation of epidemiolofical criteria for identifying outbreaks of acute gastroenteritis due to norovirus: United States, 1998-2000. Clin Infect Dis. 2006 Apr 1;42(7):9649.

5. Teunis PF, Moe CL, Liu P, Miller SE, Lindesmith L, Baric RS, et al. Norwalk virus: how infectious is it?. J Med Virol. 2008 Aug;80(8):1468-76. doi: 10.1002/jmv.21237.

6. Duizer E, Bijkerk P, Rockx B, De Groot A, Twisk F, Koopmans M. Inactivation of calicivirus. Appl Environ Microbiol. 2004 Aug;70(8):4538-43.

7. Historical perspective: norovirus gastroenteritis outbreaks in military forces. MSMR. 2011 Nov;18(11):7-8.

8. Gonzaga VE, Ramos M, Maves RC, Freeman R, Montgomery JM. Concurrent outbreak of norovirus genotype I and enterotoxigenic Escherichia coli on a U.S. Navy ship following a visit to Lima, Peru. PLoS One. 2011;6(6):e20822. doi: 10.1371/journal.pone.0020822.

9. Reaves AJ, Kasper MR, Chimelski E, Klein ML, Valle R, Edgel KA, et al.
Outbreak of gastrointestinal illness during Operation New Horizons in Pisco, Peru, July 2012. MSMR. 2012 Nov;19(11):17-9.

10. Delacour H, Dubrous P, Koeck JL. Noroviruses: a challenge for military forces. J R Army Med Corps. 2010 Dec;156(4):251-4.

11. Chapman AS, Witkop CT, Escobar JD, Schlorman CA, DeMarcus LS, Marmer LM, et al. Norovirus outbreak associated with person-toperson transmission, U.S. Air Force Academy, July 2011. MSMR. 2011 Nov; 18(11):2-5.

12. Thorton SA, Sherman SS, Farkas T, Zhong W, Torres P, Jiang X. Gastroenteritis in US Marines during operation Iraqi Freedom. Clin Infect Dis. 2005 Feb 15;40(4):519-25.

13. Saito M1, Goel-Apaza S, Espetia S, Velasquez D, Cabrera L, Loli S, et al. Multiple norovirus infections in a birth cohort in a Peruvian Periurban community. Clin Infect Dis. 2014 Feb;58(4):483-91. doi: $10.1093 / \mathrm{cid} / \mathrm{cit} 763$.

14. Ochoa TJ, Chea-Woo E, Baiocchi N, Pecho I, Campos M, Prada A, et al. Randomized double-blind controlled trial of bovine lactoferrin for prevention of diarrhea in children. J Pediatr. 2013 Feb;162(2):349-56. doi: 10.1016/j. jpeds.2012.07.043.

15. Rivera FP, Ochoa TJ, Ruiz J, Medina AM, Ecker L, Mercado E, et al. Norovirus prevalence in 'pathogen negative' gastroenteritis in children from periurban areas in Lima, Peru. Trans R Soc Trop Med Hyg. 2011 Dec;105(12):734-6. doi: 10.1016/j. trstmh.2011.08.009.

16. Yori PP, Schwab K, Gilman RH, Nappier S, Portocarrero DV, Black RE, et al. Norovirus highly prevalent cause of endemic acute diarrhea in children in the peruvian Amazon. Pediatr Infect Dis J. 2009 Sep;28(9):844-7. doi: 10.1097/INF.0b013e3181a24730.

17. Repp KK, Hostetler TP, Keene WE. A norovirus outbreak related to contaminated surfaces. J Infect Dis. 2013 Jul 15;208(2):295-8. doi: 10.1093/infdis/jit148.

18. Heyman D. Control of Communicable Disease Manual. 19th ed. Washington, DC: American Public Health Association; 2008.

19. Ramos M, Valle R, Reaves EJ, Loayza $\mathrm{L}$, Gonzalez S, Bernal $\mathrm{M}$, et al. Outbreak of Group A beta hemolytic Streptococcus pharyngitis in a Peruvian military facility, April 2012. MSMR. 2013;20(6):14-7.

20. Halperin T, Vennema H, Koopmans M, Kahila Bar-Gal G, Kayouf R, Sela T, et al. No association between histo-blood group antigens and susceptibility to clinical infections with genogroup II norovirus. J Infect Dis. 2008 Jan 1;197(1):63-5. doi: $10.1086 / 524145$.

Correspondencia: Mariana Ramos Rodriguez

Dirección: Centro Médico Naval (NAMRU-6) Av. Venezuela cuadra $36 \mathrm{~s} / \mathrm{n}$, Bellavista, Callao.

Teléfono: (+51) 949074755

Correo electrónico: mariana.ramos@med. navy.mil 